\title{
Helicenes as Chiroptical Photoswitches
}

\author{
Ravat, Prince ; Šolomek, Tomáš ; Juricek, Michal
}

\begin{abstract}
In this Concept Article, we analyze known helicene systems that function as chiroptical photoswitches from a stereochemical perspective and classify them based on the type of transformation of the helicene core that takes place during the switching process. In every case, we inspect the stereodynamics of each of the two forms of the switch and the stereospecificity of their interconversion, and we discuss the chemical working principles and chiroptical responses of the switches. We hope that our analysis will motivate the design of new types of helicene photoswitches to expand the structural diversity of this, at present rare but beautiful, class of compounds.
\end{abstract}

DOI: https://doi.org/10.1002/cptc.201800229

Posted at the Zurich Open Repository and Archive, University of Zurich ZORA URL: https://doi.org/10.5167/uzh-176884

Journal Article

Accepted Version

Originally published at:

Ravat, Prince; Šolomek, Tomáš; Juricek, Michal (2019). Helicenes as Chiroptical Photoswitches. ChemPhotoChem, 3(4):180-186.

DOI: https://doi.org/10.1002/cptc.201800229 


\section{CHEMISTRY OF LIGHT INTERACTION \\ CHEMPHOTOCHEM}

ACROSS THE WHOLE SPECTRUM

\section{Accepted Article}

Title: Helicenes as Chiroptical Photoswitches

Authors: Prince Ravat, Tomáš Šolomek, and Michal Juricek

This manuscript has been accepted after peer review and appears as an Accepted Article online prior to editing, proofing, and formal publication of the final Version of Record (VoR). This work is currently citable by using the Digital Object Identifier (DOI) given below. The VoR will be published online in Early View as soon as possible and may be different to this Accepted Article as a result of editing. Readers should obtain the VoR from the journal website shown below when it is published to ensure accuracy of information. The authors are responsible for the content of this Accepted Article.

To be cited as: ChemPhotoChem 10.1002/cptc.201800229

Link to VoR: http://dx.doi.org/10.1002/cptc.201800229 


\title{
Helicenes as Chiroptical Photoswitches
}

\author{
Prince Ravatt ${ }^{+}{ }^{[a]}$ Tomáš Šolomek $^{+},{ }^{[b]}$ and Michal Juríček ${ }^{*[c]}$ \\ Dedicated to Michel Rickhaus on the occasion of starting his independent research
}

\begin{abstract}
In this concept article, we analyze known helicene systems that function as chiroptical photoswitches from a stereochemical perspective and classify them based on the type of transformation of the helicene core that takes place during the switching process. In every case, we inspect the stereodynamics of each of the two forms of the switch, stereospecificity of their interconversion, and we discuss the chemical working principles and chiroptical responses of the switches. We hope that our analysis will motivate the design of new types of helicene photoswitches to expand the structural diversity of this, at present rare but beautiful, class of compounds.
\end{abstract}

\section{Introduction}

Helicenes ${ }^{[1]}$ are helically twisted chiral $\pi$-conjugated molecules comprised of ortho-fused benzenoid rings. They have been known for more than a century and last year marked 100 years from the time the first [5]helicene ${ }^{[2]}$ was synthesized. Since then, even helicenes as long as 16 benzenoid rings ${ }^{[3]}$ have been constructed. Helicenes display high values of optical rotation of linearly polarized light and large degrees of differential absorption of left- and right-handed circularly polarized light. This feature makes helicenes promising candidates for use in optical data storage, among other applications, ${ }^{[4]}$ which requires that helicene systems can be switched reversibly between two forms. If the two forms have distinct chiroptical properties, linearly or circularly polarized light can be used for a non-destructive read-out.

Helicene-based chiroptical switches that have been reported can be triggered either by acid/base ${ }^{[5]}$ oxidation/reduction, ${ }^{[6]}$ or light. ${ }^{[7]}$ Although light irradiation represents the least invasive trigger, switches operated by light are the least common. Despite the relatively small number of examples, photoswitchable helicenes have been reviewed ${ }^{[4]}$ on several occasions. To expand structural

[a] Dr. Prince Ravat ${ }^{+}$ Institute of Organic Chemistry University of Würzburg Am Hubland D-97074 Würzburg (Germany)

[b] Dr. Tomáš Šolomek ${ }^{+}$ Department of Chemistry University of Basel St. Johanns-Ring 19 $\mathrm{CH}-4056$ Basel (Switzerland)

[c] Prof. Michal Juríček Department of Chemistry University of Zurich Winterthurerstrasse 190 CH-8057 Zurich (Switzerland) Email: michal.juricek@chem.uzh.ch diversity and inspire the design of new types of these photochromic materials, we set out to analyze and conceptually classify the examples in the literature from a stereochemical perspective (Scheme 1). In this account, the term helicene switch refers to systems, in which the structural motif that is responsible for the switching function is integrated within the helicene core. In the case of multiple analogous systems, the most representative examples were selected and are shown to illustrate the concept. In the graphical representations, all rings to become and those that are part of the helicene core are blue-filled, except for those that are formed during the switching process.

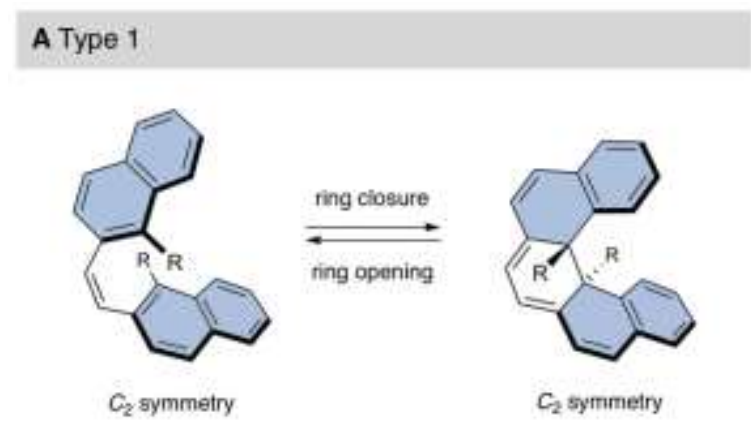

B Type 2
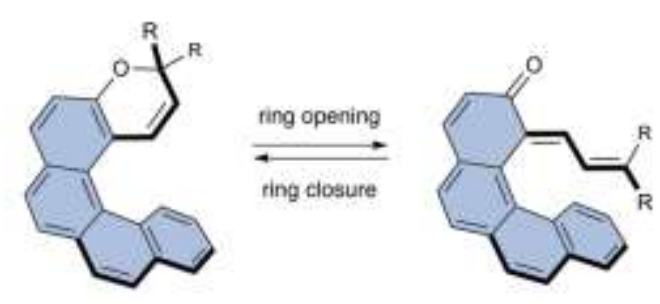

$C_{7}$ symmetry

$C_{1}$ symmetry

\section{Type 3}

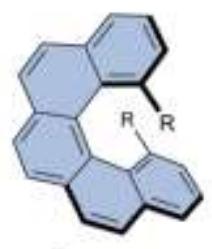

$c_{2}$ symmetry
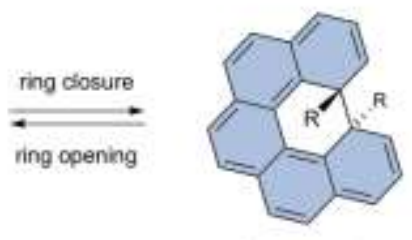

$C_{2}$ symmetry
Scheme 1. Classification of known helicene-based chiroptical photoswitches, based on the type of transformation of the helicene core that takes place during the switching process.

$\left.{ }^{+}\right]$These authors contributed equally to this work. 


\section{Classification}

From the stereochemical view, the known helicene photoswitches can be classified ${ }^{[8]}$ into three types, as shown in Scheme 1 by using [5] helicene core as an illustrative structural motif:

In type 1 systems $(A)$, the helicene core is formed during the ring closure of the diarylethene open form (left) to give the helicene closed form (right) by light. In the open form, the rotation of each aryl unit about the respective single bond is restricted, which leads to three stereoisomers that are in a dynamic equilibrium: a pair of enantiomers with an antiparallel orientation of the aryl units and an achiral stereoisomer with a parallel orientation of the aryl units (shown in Scheme 2). Under the photochemical conditions, the ring closure of the parallel form is a symmetry-forbidden process ${ }^{[9]}$ and only the antiparallel form reacts to give the helicene closed form with an anti-orientation of the $\mathrm{R}$ groups. During this transformation, each enantiomer of the antiparallel open form gives the corresponding enantiomer of the anti-closed form, which retains the "helical sense" of the diarylethene motif. While the anticonfiguration of the $\mathrm{R}$ groups in the closed form is fixed, helicene inversion can occur and give the corresponding diastereomer, depending on the activation energy of the diastereoisomerization process $^{[10]}$ and the relative energies of the diastereomers.

In type 2 systems (B), the terminal ring of the helicene core is opened during the transformation of the closed (left) to the open (right) helicene form by light. Each form can exist in one of two enantiomeric forms, where each enantiomer is transformed stereospecifically during the switching process. The helical sense is retained during this transformation, given that the activation energy of helicene inversion is sufficiently high.

In type 3 systems (C), a bond is formed between the fjord carbon atoms of the helicene core during the transformation of the open helicene form (left) to the closed form (right) by light. In the closed form, the core is partially planarized and the R groups adopt an anti-orientation. Each form can exist in one of two enantiomeric forms and during the switching process, each enantiomer of one form is transformed to the corresponding enantiomer of the other form. While type 2 and type 3 systems both operate stereospecifically, given that the activation energy of helicene inversion is sufficiently high, stereoselectivity in type 1 systems needs to be induced if the open-form stereoisomers interconvert fast at room temperature.

From the chemistry point of view, the design of helicene photoswitches has so far relied solely on $6 \pi$ electrocyclic reactions, where one $\pi$-bond is cleaved while one $\sigma$-bond is formed, and vice versa. These transformations are based either on (1) ring closure of the 1,3,5-hexatriene form and the reverse ring opening of the 1,3-cyclohexadiene form ${ }^{[11]}$ (type 1 and type 3 systems) or (2) ring opening of the $2 \mathrm{H}$-pyran form and the reverse ring closure of the 2,4-pentadienal form ${ }^{[12]}$ (type 2 systems). Typically, 1,3,5hexatriene and $2 \mathrm{H}$-pyran forms are colorless and 1,3cyclohexadiene and 2,4-pentadienal forms are colored. Photoswitching can therefore be mediated by UV light in one direction and by visible light in the reverse direction. From the perspective of thermal reversibility at room temperature, these systems can be classified as T-type or P-type. While switching of the T-type systems in one of the two directions proceeds readily also under the thermal conditions, switching of the P-type systems in both directions can only be triggered by light. From the structural standpoint, all of the known systems but one feature a heterohelicene backbone. The first example of the carbohelicene system that does not contain any heteroatoms has recently been described ${ }^{[13]}$ by our group.

\section{Type 1}

The structural motif that is responsible for the switching function in the type 1 systems is 1,2-diarylethene, which is an expansion of 1,3,5-hexatriene. As photochemical electrocyclizations of 1,2diphenylethenes often display thermal reversibility, thienyl has become ${ }^{[11]}$ the aryl group of choice for the design of P-type systems. 1,2-Dithienylethene is the only photochromic system that has so far been used in the design of type 1 photoswitch systems. Three structural variations of 1,2-dithienylethenes are possible, where the thienyl groups are connected either through $2,2^{\prime}, 3,3$ ', or 2,3' positions. While the ring closure of extended 2,2' systems generates one helicene unit, the ring closure of extended $3,3^{\prime}$ or $2,3^{\prime}$ systems can form up to two helicene units. Representative examples of 1,2-dithienylethene-based systems with 2,2' and 3,3' linkages are shown in Schemes 2 and 3, respectively.

The first example of 1,2-dithienylethene system with a 2,2' linkage, helicene-based photoswitch 1, was reported ${ }^{[14]}$ in 2001 by Branda and co-workers. By fusing one naphathalene unit to each thienyl unit, they obtained a system, where the ring closure of the open form 01 generates the closed form c1 with a [7]heterohelicene backbone (Scheme 2, A). The open form 01 is stereodynamic and the interconversion between the antiparallel- $\left(R_{\mathrm{a}}, R_{\mathrm{a}}\right)$ and $-\left(S_{\mathrm{a}}, S_{\mathrm{a}}\right)$ and the parallel- $\left(R_{\mathrm{a}}, S_{\mathrm{a}}\right)$ stereoisomers is fast at room temperature. Achiral stereoisomer $\left(R_{\mathrm{a}}, S_{\mathrm{a}}\right)$-01 is higher in energy compared with its chiral stereoisomers $\left(R_{\mathrm{a}}, R_{\mathrm{a}}\right)$-01 and $\left(S_{\mathrm{a}}, S_{\mathrm{a}}\right)$-01, and represents the intermediate on their enantiomerization pathway (A, top). Irradiation of 01 at $410 \mathrm{~nm}$ generates solely anti-c1 (A, bottom) as a mixture of two enantiomers $(M, S, S)$ and $(P, R, R)$. This composition was confirmed by the presence of a single set of signals assigned to $\mathrm{c} 1$ in the ${ }^{1} \mathrm{H}$ NMR spectrum of a sample obtained after irradiation and X-ray diffraction analysis of a racemic single crystal, where only these two enantiomers were present. This observation is in agreement with large activation energies of enantiomerization of [7]helicenes and the fact that the parallel conformer is unreactive under the irradiation conditions. In the photostationary state (PSS) reached after irradiation of 01 at $410 \mathrm{~nm}$, the $\mathbf{c 1 / 0 1}$ ratio was found to be $74: 26$. This state can be reversed upon irradiation at $>458 \mathrm{~nm}$, mediating the ring opening of $\mathbf{c 1}$ back to $\mathbf{0 1}$. Although this system could not be probed in terms of chiroptical responses because of the fast interconversion between the open-form stereoisomers preventing their separation, it demonstrates the working principle of type 1 helicene photoswitches. 


\section{A Branda, 2001}

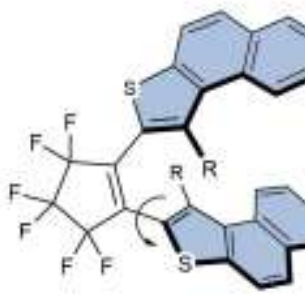

antiperallet $\left\langle R_{\mathrm{a}}, \boldsymbol{R}_{\mathrm{a}}\right\rangle-01$

$>458 \mathrm{~nm} 410 \mathrm{~nm}$

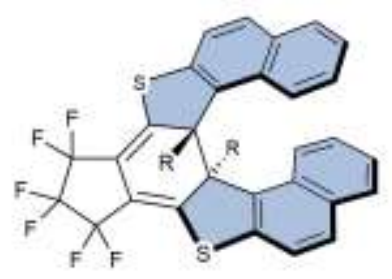

ant- $\{M, S, S\}-c 1$
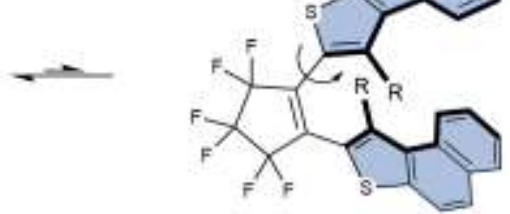

paralei- $\left(R_{a}, S_{3}\right)-01$

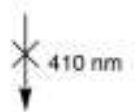

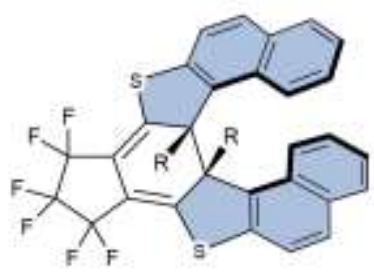

syn-(R,S)-c1

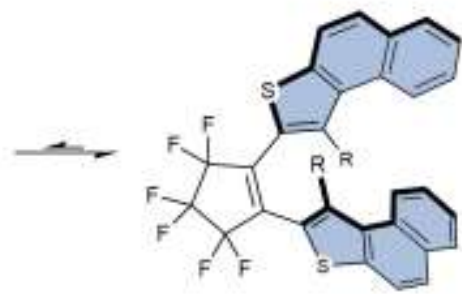

antiparaliei- $\left(S_{3}, S_{2}\right)-01$

$>458 \mathrm{~nm} 410 \mathrm{~nm}$

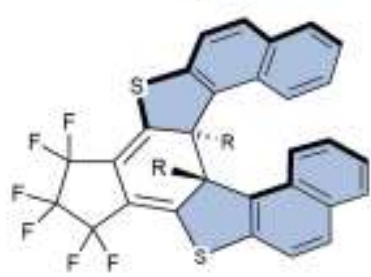

anti- $(P, A, A)-c 1$

\section{B Branda, 2005}

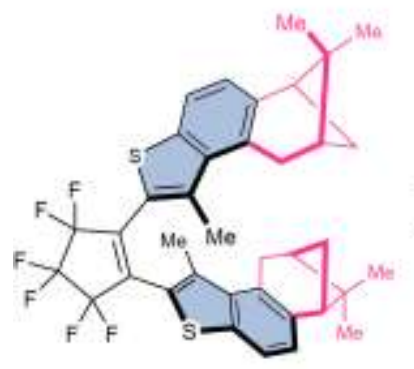

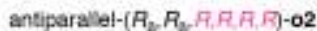

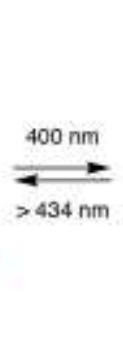

$400 \mathrm{~nm}$

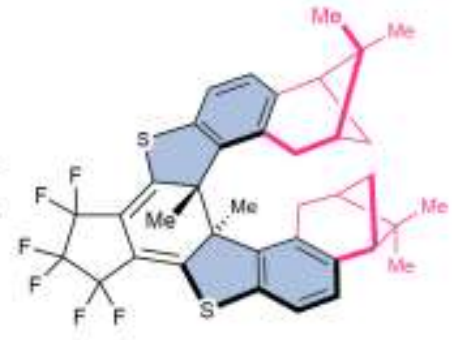

anti- $(M, S, S, R, F, F, A)-\mathrm{e} 2$

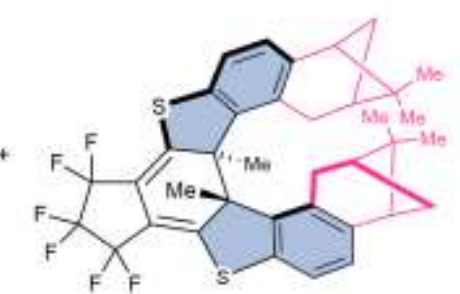

$100=0$

\section{Yokoyama, 2007}

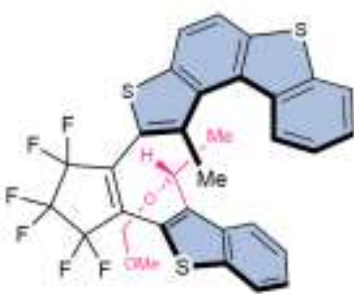

antiparallel $-\left(f_{0}, f_{\mathrm{a}}, F\right)$-03

$\underset{517 \mathrm{~nm}}{\longrightarrow}$

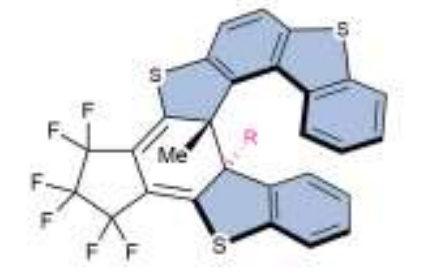

$\operatorname{ant}(M, S, S, A)-\mathrm{e} 3$

74:26

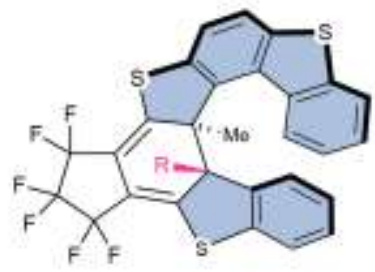

$\operatorname{arti}-(P, A, A, F)-\mathrm{c} 3$

Scheme 2. 1,2-Dithienylethene systems with a 2,2' linkage: (A) Working principle of photoswitch $1(R=M e)$, the prototype of type 1 systems developed by Branda and co-workers. (B) Branda's follow-up photoswitch system 2, where absolute diastereoselectivity was achieved by installment of two pinene units (in red) that act as chiral auxiliary groups biasing the stereochemical outcome. (C) Diastereospecific photoswitch system 3 developed by Yokoyama and co-workers, where selectivity was induced with the aid of only one chiral-auxiliary substituent $(\mathrm{R}$, in red) installed at position 3 of one of the thienyl rings. In all three cases, ring closure of the open form generates one [7]heterohelicene unit in the closed form. 
In order to turn the prototype system 1 into a chiroptical switch, chiral auxiliary groups were employed ${ }^{[15]}$ by Branda and coworkers in their follow-up work (Scheme 2, B). They obtained photoswitch 2 by replacing the two terminal benzenoid rings in $\mathbf{1}$ for two pinene units, each with an $(R, R)$ configuration. HPLC employing a chiral stationary phase performed on the open form 02 revealed the presence of only a single species. It could not, however, be determined whether this species is just one of the three possible stereoisomers, antiparallel- $\left(R_{\mathrm{a}}, R_{\mathrm{a}}, R, R, R, R\right)$-02/$\left(S_{\mathrm{a}}, S_{\mathrm{a}}, R, R, R, R\right)$-02 and parallel- $\left(R_{\mathrm{a}}, S_{\mathrm{a}}, R, R, R, R\right)$-02, or a mixture, where these stereoisomers interconvert fast at room temperature. Irradiation at $400 \mathrm{~nm}$ of this species mediating the ring closure affords a single stereoisomer of the closed form c2. Although the identity of this stereoisomer could not be confirmed by X-ray crystallography, modeling studies indicated that the more energetically favored transformation is that of antiparallel-

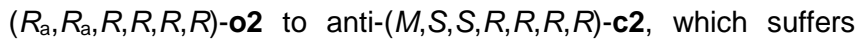
from less steric hindrance than the anti- $(P, R, R, R, R, R, R)$-c2 diastereomer (compare these two structures in Scheme 2, B). Although the c1/01 ratio $=40: 60$ in the PSS was rather disappointing, the switching could be monitored by CD and ORD spectroscopy. Notably, the difference in specific optical rotation between samples of the open form and the mixture in the PSS determined by polarimetry was as large as $9200^{\circ}$ at $365 \mathrm{~nm}$. A considerable difference of $2023^{\circ}$ was observed also at $589 \mathrm{~nm}$, where none of the two forms absorb light appreciably.

Similar chiral-auxiliary approach was adopted ${ }^{[16]}$ by Yokoyama and co-workers in their design of [7]heterohelicene-based photoswitch system 3 (Scheme 2, C). Interestingly, only one chiral-auxiliary substituent was required to bias the stereochemical outcome. Although the best solvent-dependent diastereomeric excess (de) achieved in this case was only $47 \%$, the $\mathbf{c 3 / 0 3}$ ratio in the PSS was 64:36. A difference of $1300^{\circ}$ in specific optical rotation between a sample of the open form and the mixture in the PSS was achieved at $589 \mathrm{~nm}$, where none of the two forms of $\mathbf{3}$ absorbs light. The diastereoselectivity could be improved $^{[17]}$ (up to $98 \%$ de) in analogous systems, which displayed comparable differences in specific optical rotation.

The first example of 1,2-dithienylethene system with a 3,3' linkage, helicene-based photoswitch 4, was reported ${ }^{[18]}$ in 2007 by Feringa and co-workers (Scheme 3, A). They synthesized a system, where ring closure of the open form 04 generates two [4]heterohelicene units, by fusing one biphenyl unit to the alkene double bond of 1,2-dithienylethene. In addition, high activation energy for rotation of the thienyl units each about the respective single bond allowed them, for the first time, to isolate all three stereoisomers of 04 . They could therefore demonstrate that the enantiomers of antiparallel-04 were each stereospecifically transformed into the corresponding enantiomers of the closed form anti-c4 (please note that while the anti-configuration of the methyl groups is fixed, the [4] heterohelicene units most likely undergo fast inversion). Although the absolute configuration of the enantiomers could not be determined, the switching could be monitored by CD spectroscopy. For this system, the $\mathrm{c4/04}$ ratio in the PSS and the values of optical rotation were not reported.

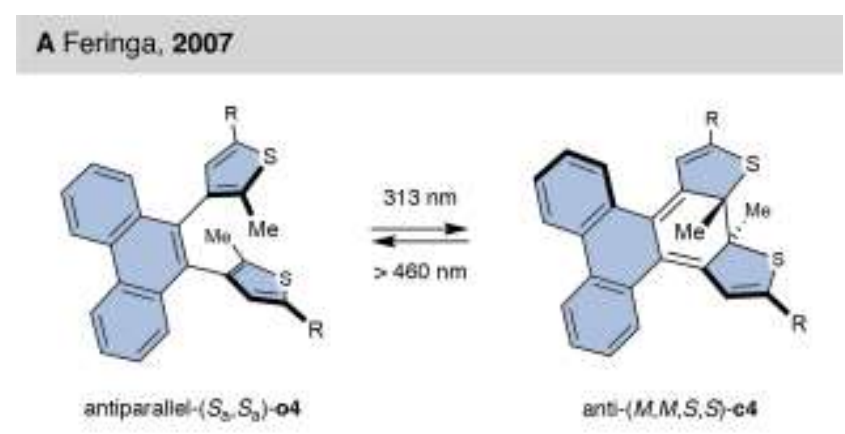

\section{B Zhu, 2015}

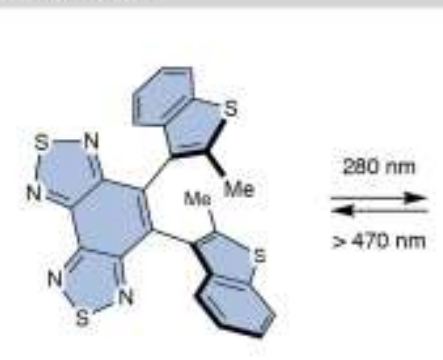

antiparallet- $\left(S_{2}, S_{2}\right)-05$

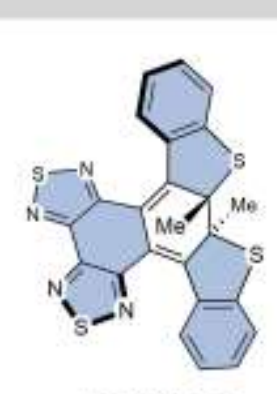

anti-\{P,P,S,S\}-e5

Scheme 3. 1,2-Dithienylethene systems with a 3,3' linkage: (A) Photoswitch system 4 developed by Feringa and co-workers, who succeeded for the first time to isolate all three stereoisomers of the open form of 1,2-dithienylethenes. (B) Photoswitch system $\mathbf{5}$ developed by Zhu and co-workers, who characterized for the first time all three stereoisomers of the open form and two stereoisomers of the closed form by means of X-ray crystallography. In both cases, ring closure of the open form generates two helicene units in the closed form.

An extended analog of 4, photoswitch $\mathbf{5}$, was developed [19] by Zhu and co-workers (Scheme 3, B). In their design, ring closure of the open form 05 generates two [5]heterohelicene units. As in the case of 04 , high activation barrier of rotation allowed them to isolate all three stereoisomers of $\mathbf{0 5}$, and each enantiomer of antiparallel-05 underwent a stereospecific ring closure to give a single enantiomer of the closed form anti-c5. Notably, all three stereoisomers of 05 and those of anti-c5 were characterized by $X$-ray crystallography, which allowed determination of absolute configurations. The formed stereoisomers were anti- $(P, P, S, S)$-c5 and anti- $(M, M, R, R)$-c5. The switching of this system, which displayed $\mathbf{c 5} / 05$ ratio $=92: 8$ in the PSS, could be monitored by CD spectroscopy and polarimetry. The difference in specific optical rotation between a sample of the open form and the mixture in the PSS was as large as $2315^{\circ}$ at $633 \mathrm{~nm}$, where none of the two forms of $\mathbf{5}$ absorbs light.

\section{Type 2}

The structural motif that is responsible for the switching function in the type 2 systems is 2,2-diphenyl-2H-chromene, ${ }^{[12]}$ which is an expansion of the $2 \mathrm{H}$-pyran motif. Incorporation of the chromene unit into a helicene backbone at one of the ends gives a system, where the helical geometry remains intact during the switching 
process. Photochemical ring opening of chromene generates the o-quinoidal open form, which has a 2,4-pentadienal unit (in red) and thus four possible stereoisomers: s-cis-cis (CC), s-cis-trans (CT), s-trans-cis (TC), and s-trans-trans (TT). The two most relevant stereoisomers, which are usually observed, ${ }^{[20]}$ are TC and TT. They can interconvert to one another thermally, but this process is relatively slow. Typically, the TC stereoisomer of the open form is thermodynamically more stable than the TT one but it undergoes a rapid thermal ring closure to the closed chromene form. As a result, ring opening of chromenes produces the TC stereoisomer as the major component of the open form and these systems operate as thermally reversible T-type photoswitches. In order for these systems to function as P-type photoswitches, the TT stereoisomer must be thermodynamically more stable than the TC stereoisomer.

This principle was first illustrated ${ }^{[21]}$ by Frigoli and co-workers in 2012 and subsequently employed ${ }^{[22]}$ by Moorthy and co-workers in 2013 by using regioisomeric chromene systems 6 and 7 . These two compounds differ in the relative orientation of the chromene unit, with the oxygen atom embedded either in the outer (6) or inner (7) rim of [6] heterohelicene (Scheme 4). In the former case, photochemical ring opening of the closed form $\mathbf{c} 6$ generates the open form 06 that undergoes a rapid thermal ring closure back to c6 within just a few minutes, which indicates that the major component of the open form is TC-06. In the latter case, the ring opening generates a single stereoisomer of the open form, namely, TT-07, which is thermally stable and undergoes thermal ring closure to the closed form $\mathbf{c 7}$ only at elevated temperatures. In this case, steric constraints imposed by the helical backbone result in higher stability of the TT stereoisomer compared with the TC one. In addition, the helical core increases the rotational barrier of the diphenylvinyl moiety in the transformation of TT-07 to TC-07, while the transformation of TC-07 to $\mathbf{~ 7 ~ i s ~ n o t ~ s t e r i c a l l y ~}$ hindered and proceeds fast. This structural feature is distinctly different from that of $\mathbf{0 6}$, where the 2,4-pentadienal moiety is integrated within the outer, and not the inner, rim of the helicene backbone. Although 07/c7 ratio in the PSS of this P-type system was only $27: 73$, it could be improved (up to 93:7) in a hexyl derivative of 7, as demonstrated ${ }^{[23]}$ by Frigoli, Jacquemin, Ortica, and co-workers. Most recently, it was shown ${ }^{[24]}$ that both forms of the hexyl derivative of $\mathbf{7}$ are stable against racemization at room temperature and that the switching of an enantioenriched sample proceeds stereospecifically and can be monitored by $C D$ spectroscopy.

\section{Type 3}

As in the case of the type 1 systems, the structural motif that is responsible for the switching function in the type 3 systems is 1,3,5-hexatriene, which is integrated into the inner rim of a helicene backbone. Photochemical ring closure of the open form gives the closed form, in which the core is almost planar and the $\mathrm{R}$ groups adopt a fixed anti-configuration. Because of the rigidity of both forms, the ring-closure and the ring-opening reactions proceed stereospecifically.

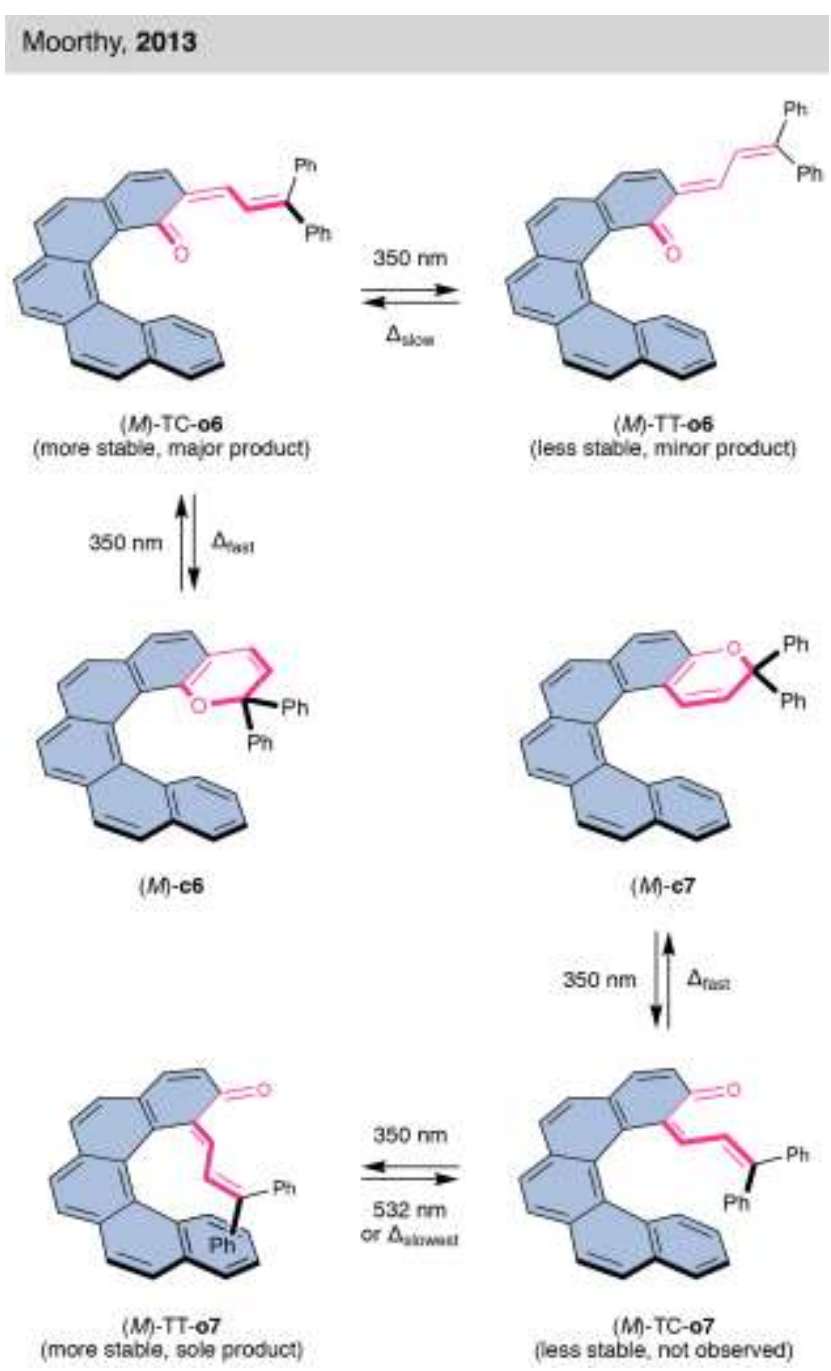

Scheme 4. 2,2-Diphenyl-2H-chromene systems 6 and 7 developed by Moorthy and co-workers, where the former system operates as a T-type and the latter one as a P-type photoswitch. In both systems, helical geometry remains intact during the switching process. The $2 \mathrm{H}$-pyran and 2,4-pentadienal units are highlighted in red.

The first type 3 system was reported ${ }^{[25]}$ in 1999 by Wang and coworkers. In their design, they integrated the 1,2-dithienylethene unit into a helical scaffold such that a [5]heterohelicene system 8 with two thiophene units as terminal rings was obtained (Scheme $5, A)$. The central ring of the [5]heterohelicene core is fused to a succinic anhydride moiety and the helicene core is partially saturated. In this system, the antiparallel orientation of the thienyl units is fixed, which prevents the interconversion between the open-form stereoisomers, an undesired feature of the type 1 systems lowering the switching efficiency. On account of the cisselectivity of the Diels-Alder reaction employed in the last step of the synthesis of $\mathbf{0 8}$, only two stereoisomers of 08 were obtained, a pair of enantiomers $(M, R, R, S, S)$-08 and $(P, R, R, S, S)$-08 (identical to $(M, S, S, R, R)$-08 and $(P, S, S, R, R)$-08, respectively). 


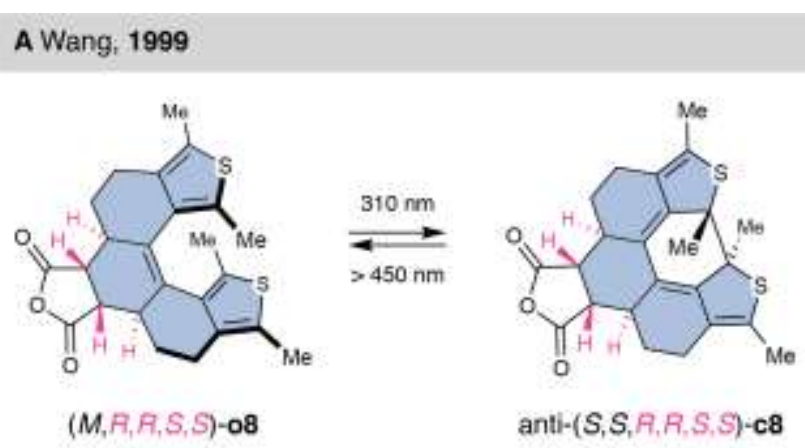

B Juriček, 2018

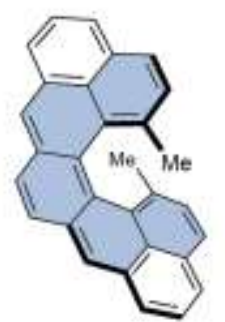

$(M)-09$

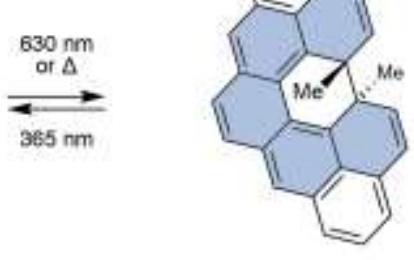

anti- $(R, F) \cdot \mathbf{c} 9$
Scheme 5. (A) 1,2-Dithienylethene system 8 with a 3,3' linkage reported by Wang and co-workers. (B) 1,2-Diphenylethene system 9 developed by our group. In both cases, the ring closure of the open [5]helicene form almost planarizes the core of the closed form.

In the photochemical ring closure of 08 , a $\sigma$-bond is formed between a pair of fjord carbon atoms each bearing a methyl substituent, a process that partially planarizes the helicene core. Although ring closure and ring opening should proceed in an stereospecific fashion, for example, $(M, R, R, S, S)$-08 would give anti-(S,S,R,R,S,S)-08 and vice versa, switching was not performed on enantioenriched samples and chiroptical responses were not studied. The $\mathbf{c 8 / 0 8}$ ratio in the PSS was also not reported.

Most recently, we reported ${ }^{[13]}$ the first example of a carbohelicene photoswitch system 9 (Scheme 5, B). The 1,3,5-hexatriene unit is integrated into the inner rim of a [5] helicene backbone in this type 3 system and two additional benzenoid rings are fused to the [5] helicene core to give a fully conjugated seven-ring system that we named ${ }^{[26]}$ cethrene. Photochemical ring closure of the open form 09 at $630 \mathrm{~nm}$ gives the closed form anti-c9, in which the methyl substituents have an anti-orientation and the core is nearly planar. The ring opening of anti-c9 is mediated by UV light at 365 $\mathrm{nm}$. On account of the small HOMO-LUMO gap in the open form, the LUMO is partially occupied ${ }^{[27]}$ in the so-called diradicaloid ground state. As a result, the activation energy of the thermal ring closure, which is formally a symmetry-forbidden process, ${ }^{[28]}$ is low and thermal ring closure to the more stable anti-c9 occurs within just a few hours at room temperature. This system therefore operates also as a T-type photoswitch. In order for this system to function exclusively as a P-type, the activation energy of the thermal process must be increased, which can be achieved by a suitable structural modification opening the HOMO-LUMO energy gap. The enantiomers of anti-c 9 were resolved by HPLC employing a chiral stationary phase, however, quantities sufficient for obtaining reliable values of specific optical rotation could not be isolated. Nonetheless, the enantiospecific nature of the switching could be validated by CD spectroscopy. The $09 / \mathrm{c} 9$ ratio in the PSS at $365 \mathrm{~nm}$ was found to be $88: 12$ and only a minor fatigue was observed in a series of consecutive all-photochemical back-and-forth cycles.

\section{Outlook}

The known examples of helicene photoswitches were classified from a stereochemical perspective into three distinct types. The majority of type 1 systems function as diastereoselective photoswitches, but a recent example illustrates that they can operate also in an enantiospecific fashion, similarly to the case of type 2 and 3 systems. Although the proof-of-concept was demonstrated in all three cases, chiroptical data are not available for all systems and conclusions as to which system is the best chiroptical switch are difficult to make at the present moment. With respect to optical rotation, type 1 and 3 systems are more promising candidates than type 2 systems, as the switching involves construction and destruction of the helicene core, which is responsible for high values of optical rotation. Other challenges that remain to be tackled include optimization of (1) stability towards degradation, (2) ratio between the open and the closed form in the PSS, (3) stereoselectivity, and (4) changes in specific optical rotation at wavelengths outside the absorption region of both forms for a non-destructive read-out. Considering the relatively small number of examples reported so far, it is crucial to expand the structural diversity of this class of materials. We hope that this concept article will motivate such endeavors.

\section{Acknowledgements}

This project received funding from the European Research Council (ERC) under the European Union's Horizon 2020 research and innovation programme (Grant Agreement No. 716139), the Swiss National Science Foundation (SNSF, T.Š./PZ00P2_174175 and M.J./PP0OP2_170534), and JuliusMaximilians-Universität Würzburg within the "Excellent Ideas" programme (P.R.).

Keywords: chiroptical properties $•$ helicene $\bullet$ photochromism $•$ stereochemistry $\cdot$ photoswitch

[1] a) M. Rickhaus, M. Mayor, M. Juríček, Chem. Soc. Rev. 2016, 45, 15421556; b) M. Gingras, Chem. Soc. Rev. 2013, 42, 968-1006; c) M. Gingras, G. Félix, R. Peresutti, Chem. Soc. Rev. 2013, 42, 1007-1050; d) R. H. Martin, Angew. Chem. Int. Ed. 1974, 13, 649-660.

[2] a) R. Weitzenböck, A. Klingler, Monatsh. Chem. 1918, 39, 315-323; b) R. Weitzenböck, A. Klingler, J. Chem. Soc. 1918, 114, 494.

[3] K. Mori, T. Murase, M. Fujita, Angew. Chem. Int. Ed. 2015, 54, 68476851. 
[4] a) C.-F. Chen, Y. Shen, Helicene Chemistry: From Synthesis to Applications, Springer, Berlin, 2017, pp. 201-220; b) H. Isla, J. Crassous, C. R. Chim. 2016, 19, 39-49; c) M. Gingras, Chem. Soc. Rev. 2013, 42, 1051-1095; d) Y. Shen, C.-F. Chen, Chem. Rev. 2012, 112, 1463-1535.

[5] For representative recent examples, see: a) P. E. Reyes-Gutiérrez, M. Jirásek, L. Severa, P. Novotná, D. Koval, P. Sázelová, J. Vávra, A. Meyer I. Císařová, D. Šaman, R. Pohl, P. Štěpánek, P. Slavíček, B. J. Coe, M. Hájek, V. Kašička, M. Urbanová, F. Teplý, Chem. Commun. 2015, 51 1583-1586; b) N. Saleh, B. Moore, II, M. Srebro, N. Vanthuyne, L. Toupet, J. A. G. Williams, C. Roussel, K. K. Deol, G. Muller, J. Autschbach, J. Crassous, Chem. Eur. J. 2015, 21, 1673-1681.

[6] For representative recent examples, see: a) D. Schweinfurth, M. Zalibera, M. Kathan, C. Shen, M. Mazzolini, N. Trapp, J. Crassous, G. Gescheidt, F. Diederich, J. Am. Chem. Soc. 2014, 136, 13045-13052; b) L. Pospišil, L. Bednárová, P. Štěpánek, P. Slaviček, J. Vávra, M. Hromadová, H. Dlouhá, J. Tarábek, F. Teplý, J. Am. Chem. Soc. 2014, 136, 1082610829.

[7] Helicene-based chiroptical switches triggered by light are reviewed in the following sections.

[8] The Cahn-Ingold-Prelog (CIP) system was used to designate absolute configurations: $R / S$ descriptors were used for $s p^{3}$-carbon atoms bearing four different substituents, $R_{a} / S_{a}$ descriptors for biaryl units, and $P / M$ descriptors for helicene units. The CIP system was employed for the nomenclature purposes only. Stereoisomerism and chirality were analyzed by inspection of the stereodynamics and overall symmetry of the molecules.

[9] a) R. B. Woodward, R. Hoffmann, The Conservation of Orbital Symmetry, Verlag Chemie, Weinheim, 1970; b) I. Fleming, Molecular Orbitals and Organic Chemical Reactions, John Wiley and Sons, Chichester, 2010 pp. 253-368; c) R. B. Woodward, R. Hoffmann, J. Am. Chem. Soc. 1965, $87,295-397$.

[10] a) J. Barroso, J. L. Cabellos, S. Pan, F. Murillo, X. Zarate, M. A. Fernandez-Herrera, G. Merino, Chem. Commun. 2018, 54, 188-191; b) P. Ravat, R. Hinkelmann, D. Steinebrunner, A. Prescimone, I. Bodoky, M. Juríček, Org. Lett. 2017, 19, 3707-3710; c) R. H. Martin, M. J. Marchant, Tetrahedron 1974, 30, 347-349; d) R. H. Martin, M. J. Marchant, Tetrahedron Lett. 1972, 13, 3707-3708.

[11] a) M. Irie, T. Fukaminato, K. Matsuda, S. Kobatake, Chem. Rev. 2014, 114, 12174-12277; b) M. Irie, Chem. Rev. 2000, 100, 1685-1716.

[12] a) J. D. Hepworth, B. M. Heron in Functional Dyes (Ed.: S.-H. Kim), Elsevier, Amsterdam, 2006, pp. 85-135; b) B. Van Gemert in Organic
Photochromic and Thermochromic Compounds, Vol. 1 (Eds.: J. C. Crano, R. J. Guglielmetti), Kluwer Academic/Plenum Publishers, New York, 1999, pp. 111-140.

[13] P. Ravat, T. Šolomek, D. Häussinger, O. Blacque, M. Juríček, J. Am. Chem. Soc. 2018, 140, 10839-10847.

[14] T. B. Norsten, A. Peters, R. McDonald, M. Wang, N. R. Branda, J. Am. Chem. Soc. 2001, 123, 7447-7448.

[15] T. J. Wigglesworth, D. Sud, T. B. Norsten, V. S. Lekhi, N. R. Branda, J. Am. Chem. Soc. 2005, 127, 7272-7273.

[16] T. Okuyama, Y. Tani, K. Miyake, Y. Yokoyama, J. Org. Chem. 2007, 72 1634-1638.

[17] a) Y. Yokoyama, T. Shiozawa, Y. Tani, T. Ubukata, Angew. Chem. Int. Ed. 2009, 48, 4521-4523; b) Y. Tani, T. Ubukata, Y. Yokoyama, Y Yokoyama, J. Org. Chem. 2007, 72, 1639-1644.

[18] M. Walko, B. L. Feringa, Chem. Commun. 2007, 1745-1747.

[19] a) W. Li, X. Li, Y. Xie, Y. Wu, M. Li, X.-Y. Wu, W.-H. Zhu, H. Tian, Sci. Rep. 2015, 5, 9186; b) W. Li, C. Jiao, X. Li, Y. Xie, K. Nakatani, H. Tian, W. Zhu, Angew. Chem. Int. Ed. 2014, 53, 4603-4607.

[20] a) Y. Kodama, T. Nakabayashi, K. Segawa, E. Hattori, M. Sakuragi, N Nishi, H. Sakuragi, J. Phys. Chem. A 2000, 104, 11478-11485; b) S. Delbaere, B. Luccioni-Houze, C. Bochu, Y. Teral, M. Campredon, G. Vermeersch, J. Chem. Soc., Perkin Trans. 2 1998, 1153-1157.

[21] M. Frigoli, F. Maurel, J. Berthet, S. Delbaere, J. Marrot, M. M. Oliveira, Org. Lett. 2012, 14, 4150-4153.

[22] a) J. N. Moorthy, S. Mandal, A. Mukhopadhyay, S. Samanta, J. Am. Chem. Soc. 2013, 135, 6872-6884; b) J. N. Moorthy, P. Venkatakrishnan, S. Sengupta, M. Baidya, Org. Lett. 2006, 8, 4891-4894.

[23] M. Frigoli, J. Marrot, P. L. Gentili, D. Jacquemin, M. Vagnini, D. Pannacci, F. Ortica, ChemPhysChem 2015, 16, 2447-2458.

[24] Y. Kim, M. Frigoli, N. Vanthuyne, N. Tamaoki, Chem. Commun. 2017, 53 , 200-203.

[25] L. Dinescu, Z. Y. Wang, Chem. Commun. 1999, 2497-2498.

[26] P. Ravat, T. Šolomek, M. Rickhaus, D. Häussinger, M. Neuburger, M. Baumgarten, M. Juriček, Angew. Chem. Int. Ed. 2016, 55, 1183-1186.

[27] a) M. Juríček, Chimia 2018, 72, 322-327; b) P. Ravat, T. Šolomek, P. Ribar, M. Juriček, Synlett 2016, 27, 1613-1617.

[28] T. Šolomek, P. Ravat, Z. Mou, M. Kertesz, M. Juriček, J. Org. Chem. 2018, 83, 4769-4774. 
WILEY-VCH

\section{Entry for the Table of Contents}

\section{CONCEPT}

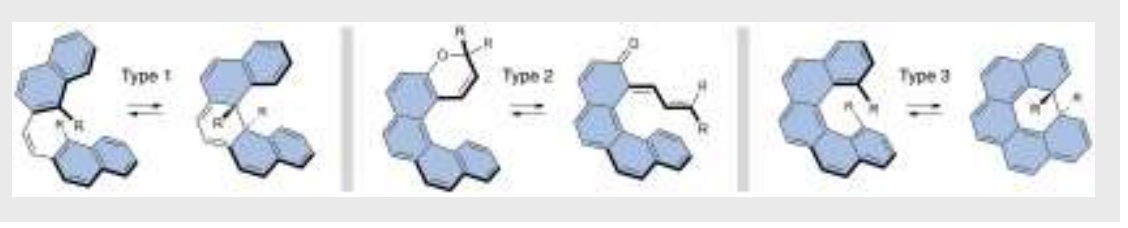

Known helicene systems that function as chiroptical photoswitches are analyzed from a stereochemical perspective. Reported examples are classified into three types based on the type of transformation of the helicene core that takes place during the switching process.
Prince Ravat, Tomáš Šolomek, and Michal Juríček*

\section{Page No. - Page No.}

Helicenes as Chiroptical Photoswitches 